\title{
Binaries discovered by the MUCHFUSS project
}

\section{FBS 0117+396: An sdB+dM binary with a pulsating primary ${ }^{\star}$}

\author{
R. H. Østensen ${ }^{1}$, S. Geier ${ }^{2,3}$, V. Schaffenroth ${ }^{3,4}$, J. H. Telting ${ }^{5}$, S. Bloemen ${ }^{1,6}$, P. Németh ${ }^{1}$, P. G. Beck ${ }^{1}$, \\ R. Lombaert ${ }^{1}$, P. I. Pápics ${ }^{1}$, A. Tillich ${ }^{3}$, E. Ziegerer ${ }^{3}$, L. Fox Machado ${ }^{7}$, S. Littlefair ${ }^{8}$, V. Dhillon ${ }^{8}$, \\ C. Aerts ${ }^{1,6}$, U. Heber ${ }^{3}$, P. F. L. Maxted ${ }^{9}$, B. T. Gänsicke ${ }^{10}$, and T. R. Marsh ${ }^{10}$ \\ ${ }^{1}$ Instituut voor Sterrenkunde, K. U. Leuven, Celestijnenlaan 200D, 3001 Leuven, Belgium \\ e-mail: roy@ster.kuleuven. be \\ 2 European Southern Observatory, Karl-Schwarzschild-Str. 2, 85748 Garching, Germany \\ 3 Dr. Karl-Remeis-Observatory \& ECAP, Astronomical Institute, F.-A. U. Erlangen-Nürnberg, 96049 Bamberg, Germany \\ ${ }^{4}$ Institute for Astro- and Particle Physics, University of Innsbruck, Technikerstr. 25/8, 6020 Innsbruck, Austria \\ 5 Nordic Optical Telescope, Rambla José Ana Fernández Pérez 7, 38711 Breña Baja, Spain \\ 6 Department of Astrophysics, IMAPP, Radboud University Nijmegen, 6500 GL Nijmegen, The Netherlands \\ 7 Observatorio Astronómico Nacional, Universidad Nacional Autónoma de México, BC 22860 Ensenada, Mexico \\ 8 Dept of Physics and Astronomy, University of Sheffield, Sheffield S3 7RH, UK \\ 9 Astrophysics Group, Keele University, Staffordshire ST5 5BG, UK \\ 10 Department of Physics, University of Warwick, Coventry CV4 7AL, UK
}

Received 5 June 2013 / Accepted 30 September 2013

\section{ABSTRACT}

\begin{abstract}
The project Massive Unseen Companions to Hot Faint Underluminous Stars from SDSS (MUCHFUSS) aims to discover subdwarf-B stars with massive compact companions such as overmassive white dwarfs $\left(M>1.0 M_{\odot}\right)$, neutron stars or black holes. From the 127 subdwarfs with substantial radial-velocity variations discovered in the initial survey, a number of interesting objects have been selected for extensive follow-up.

After an initial photometry run with BUSCA revealed that FBS $0117+396$ is photometrically variable both on long and short timescales, we chose it as an auxiliary target during a 6-night multi-color photometry run with Ultracam. Spectroscopy was obtained at a number of observatories in order to determine the binary period and obtain a radial-velocity amplitude. After establishing an orbital period of $P=0.252 \mathrm{~d}$, and removing the signal associated with the irradiated hemisphere of the M-dwarf companion, we were able to detect ten pulsation periods in the Fourier spectrum of the light curve. Two pulsation modes are found to have short periods of 337 and $379 \mathrm{~s}$, and at least eight modes are found with periods between $45 \mathrm{~min}$ and $2.5 \mathrm{~h}$. This establishes that FBS $0117+396$ is an $\mathrm{sdB}+\mathrm{dM}$ reflection binary, in which the primary is a hybrid pulsator, and the first one found with this particular mélange of flavours.
\end{abstract}

Key words. subdwarfs - surveys - binaries: close - stars: oscillations - stars: individual: FBS 0117+396

\section{Introduction}

The hot B-type subdwarf (sdB) stars are generally recognised as an extension of the horizontal branch population where the envelope mass has become too low to provide significant hydrogen burning. Binary interaction close to the tip of the red-giant branch is thought to be responsible for the vast majority of the $\mathrm{sdB}$ population, but a variety of end configurations are possible depending on the initial separation, mass and evolutionary stage of the companion (see Heber 2009, for a detailed review).

Radial-velocity surveys of sdB stars initiated by Maxted et al. (2001) and recently brought closer to completion by Copperwheat et al. (2011) find that $\sim 50 \%$ of all sdB stars reside in short-period binary systems $\left(P_{\text {orb }}<10 \mathrm{~d}\right)$. To date, more than a hundred such short-period binaries are known, and the vast majority of these have companions with masses compatible with white dwarf (WD) stars. When the companion is more massive than the subdwarf, or becomes more massive before mass transfer is terminated, the orbit will expand substantially. Such orbits

\footnotetext{
* Light curves are only available at the CDS via anonymous ftp to cdsarc.u-strasbg.fr (130.79.128.5) or via http://cdsarc.u-strasbg.fr/viz-bin/qcat?]/A+A/559/A35
}

are hard to measure, but the companion can be detected spectroscopically or from infra-red excess. Lisker et al. (2005) found that about a third of the sdBs in their survey sample show spectroscopic signatures of main-sequence (MS) companions, while Reed \& Stiening (2004), using 2MASS photometry, inferred that about half of the sdBs in the field have MS companions. In a recent development, a number of such binaries have been reported to have orbital periods extending up to 1300 days (Østensen \& Van Winckel 2011, 2012; Deca et al. 2012; Barlow et al. 2012; Vos et al. 2012). The distribution of orbital periods in both the short and long-period binaries are essential for establishing constraints on the parameters that govern the mass transfer of theoretical models, and further systems are still sought in order to improve the statistics and overcome selection biases.

The MUCHFUSS project was designed to explore the highmass end of the short-period population by pre-selecting stars from the SDSS survey (Stoughton et al. 2002) that appeared to have unusually high radial velocities. The survey strategy and sample was introduced in Geier et al. (2011a, Paper I), and seven new short-period systems were presented in Geier et al. (2011b, Paper II). A new eclipsing system in which the sdB is accompanied by a substellar companion $\left(M=0.068 M_{\odot}\right)$ with a very 
short orbital period $(P=0.096 \mathrm{~d})$ was also announced by Geier et al. (2011c, Paper III). In this paper we describe a new binary showing evidence of pulsations from the sdB primary superimposed on the strong irradiation effect from the tidally locked $\mathrm{M}$-dwarf companion. The $\mathrm{sdB}+\mathrm{dM}$ binaries constitute a fraction of the known short period binaries. Of the 89 systems listed in Appendix A of Paper I only 19 are of the sdB+dM type, and all of them have periods of less than 0.5 days (19 of 39). But the statistics from this sample are clearly biased towards those with the shortest orbital periods, as both photometric and radialvelocity variations become harder to detect for low-mass companions as the period increases.

Subdwarf-B stars have been found to pulsate, first in rapid pressure $(p$-)modes with periods between $\sim 1$ and 10 min by Kilkenny et al. (1997), and later in slower gravity $(g$-)modes with periods between $\sim 1 / 2$ and 2 h by Green et al. (2003). Such pulsators are commonly referred to as $\mathrm{sdB}$ pulsators (sdBVs), and several flavours are recognised. Conventionally, we designate pulsating stars according to the variable star names of the prototypical objects, so that the short-period pulsators are known as V361-Hya stars, the long-period pulsators are V1093Her stars, and hybrid stars displaying both types of pulsations simultaneously are known as DW-Lyn stars. Of the sdB+dM binaries, only a handful are known to have pulsating primaries. The most well known is NY Vir (Kilkenny et al. 1998), which being an eclipsing system permits one to compare detailed constraints obtained from analysing orbital spectroscopic and photometric effects (Vučković et al. 2007) with mass and radius estimates obtained from asteroseismic modelling of the pulsator (Charpinet et al. 2008; Van Grootel et al. 2013). Another one of the classic sdBV stars, V1405 Ori (Koen et al. 1999), was recently discovered to be the second $\mathrm{sdBV}+\mathrm{dM}$ binary by Reed et al. (2010b), and HE 0230-4323 was reported to be a shortperiod sdB pulsator with a strong reflection effect by Kilkenny et al. (2010). Up to now these three were the only examples of short-period sdB pulsators with M-dwarf companions. And for long-period sdBVs only a single case had been reported; JL 87 (Koen 2009), until the recent advent of space-based photometry of sdB stars. A sample of compact-pulsator candidates was monitored with the Kepler spacecraft (Østensen et al. 2010c, 2011b), and revealed a number of long-period sdBV stars with M-dwarf companions, all of which have pulsation amplitudes too low to be easily detected from the ground. The most spectacular one is 2M1938+4603 (Østensen et al. 2010a), which is an eclipsing binary with a period of $0.126 \mathrm{~d}$, in which the primary pulsates with an unusually high number of low-amplitude modes covering both long and intermediate period ranges. Two non-eclipsing $\mathrm{sdB}+\mathrm{dM}$ binaries in which the primaries are long-period pulsators of the V1093-Her type were presented by Kawaler et al. (2010a), revealing orbital periods of 0.443 and 0.395 days, a range which is difficult to discover with ground based observations. Recently, a fourth $\mathrm{sdB}+\mathrm{dM}$ binary with a pulsating primary was revealed in Kepler data by Pablo et al. (2011). This system with an orbital period of $0.399 \mathrm{~d}$ is very similar to the two systems presented by Kawaler et al. (2010a), but is a member of the open cluster NGC 6791. Interestingly, Pablo et al. (2011) were able to infer a rotation period of 9.63 days of the primary from the characteristic even splitting of the pulsation periods, thereby demonstrating that the rotation of the primary is not synchronised with the orbital period. More recently Pablo et al. (2012) have demonstrated that the same is the case for both of the long-period pulsators of Kawaler et al. (2010a). Long-period pulsations have also been reported from ground-based studies of the $\mathrm{sdB}+\mathrm{dM}$ binary GALEX J0321+4727, which has an orbital period of $0.266 \mathrm{~d}$ (Kawka et al. 2012). Since the exceptionally rich pulsation spectrum of $2 \mathrm{M} 1938+4603$ can only be explained if the $\mathrm{sdB}$ primary is rotating with a period close to the orbital, it is clear that tidal synchronisation of the primary in $\mathrm{sdB}+\mathrm{dM}$ binaries is efficient at periods of $1 / 8 \mathrm{~d}$ but not at $2 / 5 \mathrm{~d}$. The study of pulsators in systems with intermediate periods, say around $1 / 4$ to $1 / 3 \mathrm{~d}$ is therefore of interest in order to determine at which point the tidal influence becomes sufficient to enforce synchronisation. The system presented here, FBS $0117+396$, has an orbital period of $1 / 4 \mathrm{~d}$, and may therefore be significant in this respect.

\section{Discovery data}

FBS 0117+396 occurs in the Sloan Digital Sky Survey as SDSS J012022.94+395059.4 with ugriz magnitudes 15.051(4), 15.158(3), 15.638(4), 15.977(4), 16.265(7). It was originally designated FBS B12 in the First Byurakan Sky Survey (Abrahamian et al. 1990) and classified as a hot subdwarf ( $\mathrm{sdB}-\mathrm{O})$. It is close to the confusion limit of $2 \mathrm{MASS}$, and it is listed with $J$ and $K$ magnitudes of 16.02(8), and 15.9(2) and only an upper limit on $K_{\mathrm{s}}$ (Skrutskie et al. 2006). FBS 0117+396 is also found in the GALEX survey, and the magnitudes from the medium imaging survey are FUV, NUV $=14.422(6)$, 14.586(4). The extinction towards infinity in the direction of FBS $0117+396(l, b=128.9494,-22.6839)$ according to the dust maps of Schlegel et al. (1998) is $E(B-V)=0.053$.

The SDSS spectroscopic archive contains observations of this target at two epochs, the first on December 12, 2004 $(\mathrm{MJD}=53351)$ and again one year later. We downloaded the reduced SDSS 2D bundles of spectra for the two plates, and found that the first one (plate 2043) contains three individual $900 \mathrm{~s}$ integrations, and the second (plate 2336) contains five integrations with exposure times varying from 900 to $1500 \mathrm{~s}$, indicating that the last plate was taken in rather poor conditions. Still, since our target is one of the brightest on the plate, the $\mathrm{S} / \mathrm{N}$ is excellent $(>50)$ in all the individual integrations. From these eight spectra we found radial velocities that varied from close to zero to more than $100 \mathrm{~km} \mathrm{~s}^{-1}$ between the two epochs, and a trend of more than $30 \mathrm{~km} \mathrm{~s}^{-1}$ in the second epoch run, clear evidence that FBS $0117+396$ is a short-period binary. It was therefore flagged as a priority target for follow-up (see Paper I), and observed with the ISIS spectrograph on the William Herschel Telescope on La Palma, Canary Islands, in August 2009. We observed it again with WHT/ISIS a year later to further constrain the orbit solution.

In September 2010 a $2.5 \mathrm{~h}$ run of FBS $0117+396$ was obtained with the four-channel CCD camera BUSCA (Reif et al. 1999), mounted on the CAHA 2.2-m telescope. The multi-colour light curve is shown in Fig. 1, and the corresponding Fourier transform in Fig. 2. Note that BUSCA observations are done without any filters, but with passbands defined by the dichroics which splits the input beam roughly at 4400,5400 and $7300 \AA$, providing four channels designated as $u, b, r, i$ in Fig. 1 . It is immediately clear from the photometry that the target is a rapid pulsator with a pulsation semiamplitude of about $1 \%$ at a period of $\sim 340 \mathrm{~s}$, as reported briefly by Geier et al. (2012). The pulsations appear to have a higher amplitude at shorter wavelengths, as one would expect for low-order pulsation modes in sdBVs (Aerts et al. 2010). One can also notice that there is a long-term trend with a minimum during the run, which is most significant in the $i$-band, and practically absent in $u$. This is consistent with an irradiation effect in a close $s \mathrm{~dB}+\mathrm{dM}$ binary.

In order to confirm these suspicions and get a firm orbital period and velocity amplitude for the binary, we targeted 
Table 1. Log of observations.

\begin{tabular}{lllccl}
\hline \hline Year/Month/Days & Telescope & Instrument & Passbands & Resolution & P.I., Observer \\
\hline $2004 / 12 / 12$ & SDSS & Spectro & $3800-9200$ & $\sim 2000$ & Archive data \\
$2005 / 12 / 08$ & SDSS & Spectro & $3800-9200$ & $\sim 2000$ & Archive data \\
$2009 / 08 / 27-28$ & WHT $4.2 \mathrm{~m}$ & ISIS & $3700-5000$ & $\sim 3400$ & SG, AT \\
$2010 / 08 / 28-31$ & WHT $4.2 \mathrm{~m}$ & ISIS & $3700-5000$ & $\sim 2400$ & CA, RHØ \\
$2011 / 09 / 17-22$ & NOT $2.5 \mathrm{~m}$ & ALFOSC & $3500-5100$ & $\sim 2200$ & JHT, Service \\
$2011 / 11 / 15-17$ & CAHA $3.5 \mathrm{~m}$ & TWIN & $3700-5400$ & $\sim 4000$ & SG \\
$2011 / 11 / 20-21$ & NOT $2.5 \mathrm{~m}$ & ALFOSC & $3500-5100$ & $\sim 2200$ & JHT, Service \\
\hline $2010 / 09 / 27$ & CAHA $2.2 \mathrm{~m}$ & BUSCA & $u, b, r, i$ & & SG, VS \& EZ \\
$2011 / 08 / 13-14$ & Mercator $1.2 \mathrm{~m}$ & Merope II & White light & & RHØ, PP \\
$2011 / 08 / 22$ & Mercator $1.2 \mathrm{~m}$ & Merope II & White light & & RHØ, RL \\
$2011 / 08 / 28-09 / 03$ & Mercator $1.2 \mathrm{~m}$ & Merope II & White light & & RHØ, PB \\
$2011 / 08 / 16-21$ & WHT $4.2 \mathrm{~m}$ & Ultracam & $u, g, r$ & & CA, RH \& SL \\
$2011 / 08 / 27-29$ & OAN-SPM $0.84 \mathrm{~m}$ & Mexman & White light & & LFM \\
\hline
\end{tabular}

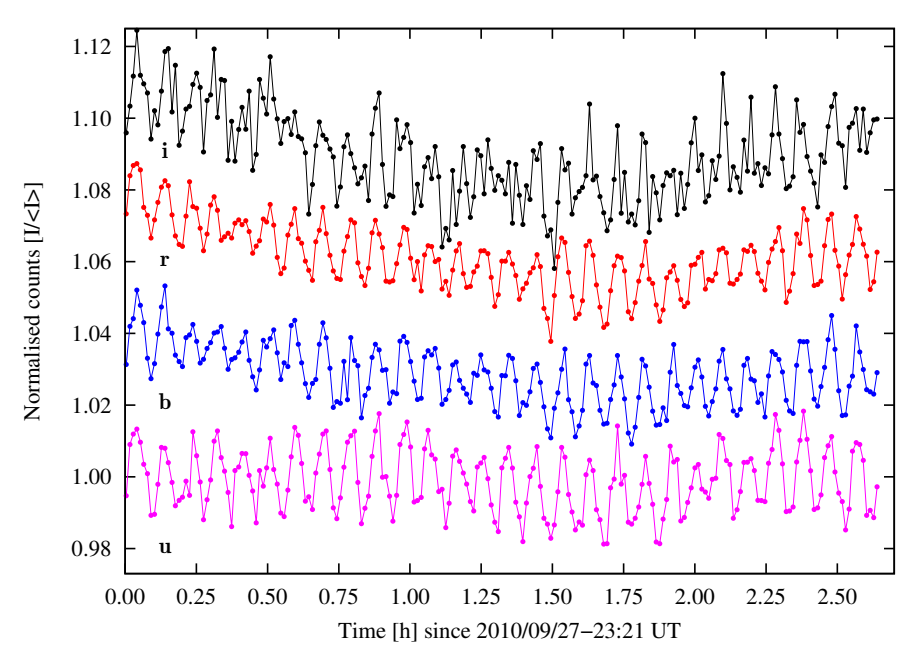

Fig. 1. Discovery light curve of FBS 0117+396 obtained with BUSCA. The $b, r$ and $i$ curves have been shifted upward by $0.03 n$ for clarity.

FBS 0117+396 at several opportunities in 2011. Service-mode observations netting 17 spectra were obtained on two occasions with ALFOSC on the 2.5-m Nordic Optical Telescope (NOT), also on La Palma, and another 17 spectra were obtained with the TWIN spectrograph on the 3.5-m telescope at the GermanSpanish Astronomical Center at Calar Alto (CAHA) in Spain. Details for these observations are given in the upper half of Table 1.

During a photometric campaign on the peculiar lowgravity sdB pulsator GALEX J201337+092801 (Østensen et al. 2011a) in August 2011, we took the opportunity to observe FBS $0117+396$ as a secondary target when the priority target started to get low in the sky. The photometric observations are listed in the lower half of Table 1, and will be analysed in detail in Sect. 4.

\section{Spectroscopic follow-up observations}

The spectroscopic observations from WHT, CAHA and NOT were extracted and processed with standard IRAF ${ }^{1}$ tasks. Radial velocities were computed with fxcor, by cross-correlating with a synthetic template derived from a suitable mean spectrum of

\footnotetext{
1 IRAF is distributed by the National Optical Astronomy Observatory; see http://iraf.noao.edu/
}

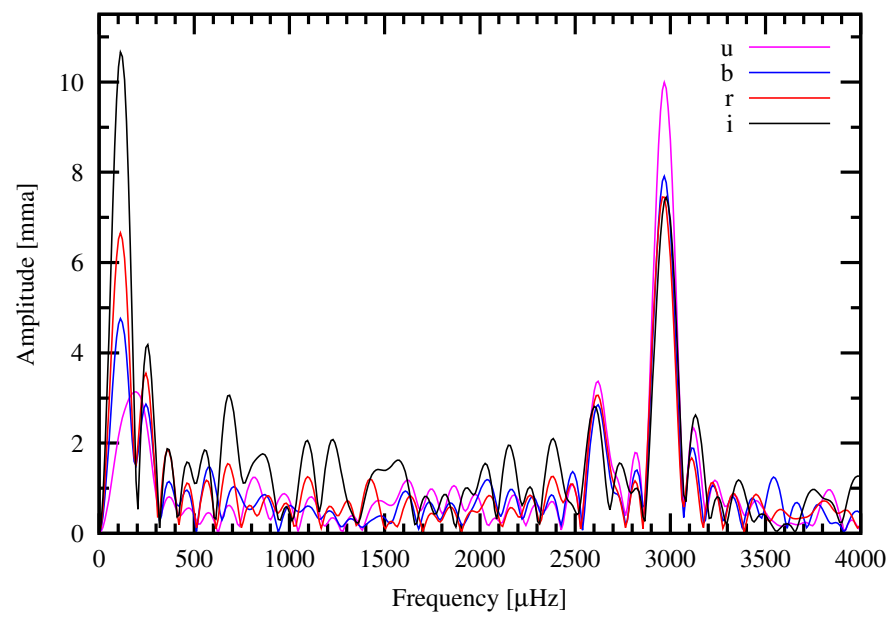

Fig. 2. Amplitude spectrum of the discovery light curves obtained with BUSCA, as shown in Fig. 1.

the target. During the processing we encountered a problem with the calibrations for some of the WHT data. It turned out that for some of the runs, instead of obtaining an arc at the beginning and at the end of the sequence, in order to calibrate the wavelength solution as interpolations of the two arcs, for the sequences obtained in 2009 only a single arc was taken close to the middle of each set, and for the last sequence taken in 2010, only a single arc was obtained at the beginning. When checking the position of the interstellar Ca II K-line at $3933 \AA$, it was found to move by more than $30 \mathrm{~km} \mathrm{~s}^{-1}$ over sequences lasting about one hour, much more than anticipated. Fortunately, our spectra have fairly high $\mathrm{S} / \mathrm{N}$ and the K-line is quite strong and can be measured quite reliably. We therefore computed the position of the K-line for each spectrum, and for each set of observations we computed either an average velocity correction from the whole set (for those with good wavelength solutions), or a linear fit (for those with wavelength calibration problems). Note that this correction with respect to an insterstellar line circumvents the need for a heliocentric correction, but increases the uncertainty of each measurement by the error on the K-line measurement. In Table 2 the radial velocities are listed for each spectroscopic observation as measured by the crosscorrelation procedure $\left(\mathrm{RV}_{\text {obs }}\right)$ together with their values as corrected with the heliocentric velocity $\left(R V_{\text {hel }}\right)$, and with respect to the $\mathrm{K}$-line $\left(\mathrm{RV}_{\mathrm{K}}\right)$ when taken to be constant at its mean value of 
Table 2. Radial velocity data.

\begin{tabular}{|c|c|c|c|c|c|c|c|c|c|c|c|}
\hline $\begin{array}{l}\text { HJD } \\
-2450000\end{array}$ & $\begin{array}{c}\mathrm{RV}_{\mathrm{obs}} \\
{\left[\mathrm{km} \mathrm{s}^{-1}\right]}\end{array}$ & $\begin{array}{c}\mathrm{RV}_{\mathrm{hel}} \\
{\left[\mathrm{km} \mathrm{s}^{-1}\right]}\end{array}$ & $\begin{array}{c}\mathrm{RV}_{\mathrm{K}} \\
{\left[\mathrm{km} \mathrm{s}^{-1}\right]}\end{array}$ & $\begin{array}{c}\sigma_{\mathrm{RV}} \\
{\left[\mathrm{km} \mathrm{s}^{-1}\right]}\end{array}$ & $\begin{array}{c}\sigma_{\mathrm{K}} \\
{\left[\mathrm{km} \mathrm{s}^{-1}\right]}\end{array}$ & $\begin{array}{l}\text { HJD } \\
-2450000\end{array}$ & $\begin{array}{c}\mathrm{RV}_{\mathrm{obs}} \\
{\left[\mathrm{km} \mathrm{s}^{-1}\right]}\end{array}$ & $\begin{array}{c}\mathrm{RV}_{\text {hel }} \\
{\left[\mathrm{km} \mathrm{s}^{-1}\right]}\end{array}$ & $\begin{array}{c}\mathrm{RV}_{\mathrm{K}} \\
{\left[\mathrm{km} \mathrm{s}^{-1}\right]}\end{array}$ & $\begin{array}{c}\sigma_{\mathrm{RV}} \\
{\left[\mathrm{km} \mathrm{s}^{-1}\right]}\end{array}$ & $\begin{array}{c}\sigma_{\mathrm{K}} \\
{\left[\mathrm{km} \mathrm{s}^{-1}\right]}\end{array}$ \\
\hline 3351.57631 & 5.01 & -14.74 & -14.74 & 19.18 & 8.66 & 5439.65877 & -15.02 & 6.50 & -87.92 & 11.80 & 2.00 \\
\hline 3351.58874 & 11.71 & -8.07 & -8.07 & 18.92 & 8.66 & 5439.66930 & -14.47 & 7.02 & -66.79 & 10.34 & 2.00 \\
\hline 3351.60131 & 3.82 & -15.99 & -15.99 & 19.85 & 8.66 & 5439.67983 & -23.42 & -1.95 & -55.16 & 11.18 & 2.00 \\
\hline 3712.57476 & -86.28 & -104.58 & -75.32 & 20.76 & 6.70 & 5439.69036 & -35.20 & -13.75 & -47.12 & 7.43 & 2.00 \\
\hline 3712.59380 & -85.61 & -103.95 & -74.69 & 20.60 & 6.70 & 5822.45519 & -101.27 & -84.69 & -82.70 & 7.77 & 5.50 \\
\hline 3712.61486 & -68.61 & -86.99 & -57.73 & 20.50 & 6.70 & 5822.48965 & -64.93 & -48.40 & -46.36 & 6.62 & 5.50 \\
\hline 3712.63068 & -61.57 & -79.99 & -50.73 & 21.04 & 6.70 & 5822.51908 & -34.36 & -17.89 & -15.79 & 6.91 & 5.50 \\
\hline 3712.64250 & -51.59 & -70.04 & -40.78 & 21.19 & 6.70 & 5822.53630 & -41.10 & -24.66 & -22.53 & 7.39 & 5.50 \\
\hline 5070.66930 & -82.62 & -60.27 & -83.11 & 10.67 & 1.63 & 5826.56409 & -18.80 & -3.89 & 1.29 & 8.27 & 5.50 \\
\hline 5070.67651 & -86.93 & -64.59 & -80.56 & 10.70 & 1.63 & 5826.60696 & -24.50 & -9.69 & -4.41 & 13.31 & 5.50 \\
\hline 5070.68372 & -86.34 & -64.02 & -73.11 & 11.98 & 1.63 & 5826.65140 & -62.10 & -47.40 & -42.01 & 9.59 & 5.50 \\
\hline 5070.69424 & -93.34 & -71.04 & -70.96 & 8.45 & 1.63 & 5881.38563 & -65.27 & -74.54 & -95.78 & 15.03 & 3.05 \\
\hline 5070.70063 & -94.55 & -72.27 & -65.31 & 9.24 & 1.63 & 5881.39530 & -77.99 & -87.29 & -108.50 & 16.16 & 3.05 \\
\hline 5070.70784 & -101.39 & -79.12 & -65.29 & 6.18 & 1.63 & 5881.40882 & -55.42 & -64.73 & -85.93 & 12.81 & 3.05 \\
\hline 5071.53483 & -12.35 & 10.04 & -24.27 & 7.65 & 1.63 & 5881.41086 & -45.95 & -55.28 & -76.46 & 19.12 & 3.05 \\
\hline 5071.54204 & -10.57 & 11.81 & -16.39 & 8.63 & 1.63 & 5881.42160 & -62.24 & -71.60 & -92.75 & 16.95 & 3.05 \\
\hline 5071.54925 & -9.84 & 12.52 & -9.56 & 10.38 & 1.63 & 5881.43146 & -59.12 & -68.50 & -89.63 & 12.58 & 3.05 \\
\hline 5071.56058 & -15.73 & 6.62 & -6.31 & 11.47 & 1.63 & 5881.43953 & -40.86 & -50.26 & -71.37 & 18.59 & 3.05 \\
\hline 5071.56779 & -24.13 & -1.80 & -8.61 & 8.52 & 1.63 & 5881.44752 & -40.61 & -50.02 & -71.12 & 11.91 & 3.05 \\
\hline 5071.57500 & -35.19 & -12.87 & -13.57 & 7.52 & 1.63 & 5881.45686 & -23.46 & -32.90 & -53.97 & 17.45 & 3.05 \\
\hline 5071.63409 & -61.12 & -38.91 & -63.13 & 6.84 & 4.00 & 5881.46488 & -12.73 & -22.18 & -43.24 & 15.40 & 3.05 \\
\hline 5071.64477 & -81.25 & -59.07 & -80.21 & 8.09 & 4.00 & 5881.56221 & -1.18 & -10.82 & -31.69 & 16.12 & 3.05 \\
\hline 5436.62102 & -66.71 & -44.42 & -73.29 & 9.41 & 1.41 & 5881.57240 & -18.53 & -28.18 & -49.04 & 12.41 & 3.05 \\
\hline 5436.63085 & -65.85 & -43.58 & -72.43 & 7.60 & 1.41 & 5882.52260 & 5.31 & -4.71 & -25.20 & 10.98 & 3.05 \\
\hline 5436.64138 & -55.27 & -33.02 & -61.85 & 9.38 & 1.41 & 5882.54198 & 17.34 & 7.29 & -13.17 & 8.85 & 3.05 \\
\hline 5436.65191 & -43.17 & -20.94 & -49.75 & 8.78 & 1.41 & 5882.55276 & 41.87 & 31.80 & 11.36 & 15.48 & 3.05 \\
\hline 5436.66244 & -34.20 & -11.99 & -40.78 & 10.70 & 1.41 & 5882.56747 & -7.66 & -17.75 & -38.17 & 11.11 & 3.05 \\
\hline 5436.67430 & -26.62 & -4.45 & -33.20 & 10.07 & 1.41 & 5886.33142 & -13.73 & -25.16 & -11.93 & 7.46 & 3.89 \\
\hline 5436.68482 & -22.20 & -0.04 & -28.78 & 8.62 & 1.41 & 5886.39885 & -66.14 & -77.40 & -64.34 & 9.89 & 3.89 \\
\hline 5436.69535 & -14.05 & 8.08 & -20.63 & 7.07 & 1.41 & 5886.45854 & -62.83 & -74.24 & -61.03 & 7.53 & 3.89 \\
\hline 5436.70588 & -5.54 & 16.57 & -19.75 & 6.83 & 4.00 & 5886.50125 & -30.91 & -42.46 & -29.11 & 10.41 & 3.89 \\
\hline 5437.69600 & -11.23 & 10.68 & -32.30 & 9.88 & 4.00 & 5886.54449 & -6.50 & -18.15 & -4.70 & 11.38 & 3.89 \\
\hline 5438.71102 & -4.38 & 17.26 & -36.88 & 6.51 & 2.31 & 5886.58586 & -1.74 & -13.49 & 0.06 & 12.86 & 3.89 \\
\hline 5438.72154 & -4.85 & 16.77 & -20.58 & 6.10 & 2.31 & 5886.62683 & -47.49 & -59.32 & -45.69 & 13.34 & 3.89 \\
\hline 5438.73207 & -9.91 & 11.69 & -8.87 & 6.59 & 2.31 & 5886.67472 & -78.84 & -90.73 & -77.04 & 11.96 & 3.89 \\
\hline 5439.65877 & -15.02 & 6.50 & -87.92 & 11.80 & 2.00 & & & & & & \\
\hline
\end{tabular}

3933.064 $\AA$. The errors listed as $\sigma_{\mathrm{RV}}$ and $\sigma_{\mathrm{K}}$ in Table 2 are respectively VERR from the IRAF fxcor task and the RMS error reported when fitting a Gaussian profile to the K-line with the Levenberg-Marquardt fitting method built into the IRAF splot task.

The best fitting solution, assuming a circular orbit, for the $\mathrm{K}$-line corrected set of velocities is:

$$
\begin{aligned}
T_{0} & =2455439.6843 \pm 0.0022 \mathrm{~d} \\
P & =0.252013 \pm 0.000013 \mathrm{~d} \\
\gamma & =--47.3 \pm 1.3 \mathrm{~km} \mathrm{~s}^{-1} \\
K_{1} & =37.3 \pm 2.8 \mathrm{~km} \mathrm{~s}^{-1}
\end{aligned}
$$

where $T_{0}$ is the Heliocentric Julian Date (HJD) of zero phase (where the primary is at the closest point to the observer), $P$ is the orbital period, $\gamma$ is the velocity of the binary system relative to the sun, and $K_{1}$ is the semi-amplitude of the radial velocity variation.

The mass function for this system is then

$f(m)=\frac{\left(M_{2} \sin i\right)^{3}}{\left(M_{1}+M_{2}\right)^{2}}=0.00135 M_{\odot}$,

which implies that for a typical $M_{1}=0.47 M_{\odot}$, and a secondary at the substellar limit $\left(M_{2}=0.075 M_{\odot}\right)$, the inclination angle must be $i=80^{\circ}$, which is ruled out by the absence of eclipses. A more massive secondary would imply a lower angle, i.e. a mass of $0.1 M_{\odot}$ corresponds to $i=50^{\circ}$, which is permitted.

\subsection{Physical parameters}

We constructed mean spectra for each run by shifting the observed spectra according to the determined orbit. The hydrogen and helium lines of each such detrended mean spectrum were then fitted with a grid of synthetic spectra calculated from fully line blanketed LTE model atmospheres assuming solar metalicity (Heber et al. 2000). The results of these fits are listed in Table 3. Note that formal fitting errors stated in the table do not account for systematic effects inherent in the models, so we generously increase the errors when stating $T_{\text {eff }}=28500 \pm 500 \mathrm{~K}$, $\log g=5.42 \pm 0.10$, and $\log \left(N_{\mathrm{He}} / N_{\mathrm{H}}\right)=-3.05 \pm 0.10$.

We also made a fit of the mean WHT spectrum from 2010 using the TLUSTY code (Hubeny \& Lanz 1995), which is a general NLTE model atmosphere code for early-type stars, treating metal line blanketing by opacity sampling. We applied the XTGRID fitting program (Németh et al. 2012), and including the first 30 elements in the fit. The fitting algorithm is a standard $\chi^{2}$-minimization technique, where the procedure starts from a detailed model and with successive approximations along the steepest gradient of the $\chi^{2}$ surface, it converges on a solution. The procedure was designed to fit the entire spectrum and not 
Table 3. Physical parameters derived from the detrended mean spectra.

\begin{tabular}{lccc}
\hline \hline Spectrum & $\begin{array}{c}T_{\text {eff }} \\
{[\mathrm{kK}]}\end{array}$ & $\begin{array}{c}\log g \\
{[\mathrm{dex}]}\end{array}$ & $\begin{array}{c}\log y \\
{[\mathrm{dex}]}\end{array}$ \\
\hline SDSS & $27.8 \pm 0.3$ & $5.32 \pm 0.03$ & $-3.01 \pm 0.07$ \\
WHT1 & $27.9 \pm 0.1$ & $5.45 \pm 0.01$ & $-3.03 \pm 0.04$ \\
WHT2 & $28.6 \pm 0.1$ & $5.43 \pm 0.01$ & $-3.02 \pm 0.02$ \\
NOT1 & $28.5 \pm 0.2$ & $5.42 \pm 0.03$ & $-3.01 \pm 0.06$ \\
CAHA & $28.7 \pm 0.3$ & $5.41 \pm 0.03$ & $-3.04 \pm 0.10$ \\
NOT2 & $28.8 \pm 0.3$ & $5.42 \pm 0.04$ & $-2.99 \pm 0.07$ \\
\hline Adopted & $28.5 \pm 0.1$ & $5.42 \pm 0.01$ & $-3.02 \pm 0.05$ \\
\hline
\end{tabular}

Table 4. Fitted lines with equivalent widths larger then $50 \mathrm{~m} \AA$.

\begin{tabular}{ccc|lcc}
\hline \hline Ion & $\begin{array}{c}\text { Wavelength } \\
{[\AA]}\end{array}$ & $\begin{array}{c}W_{\lambda} \\
{[\mathrm{m} \AA]}\end{array}$ & Ion & $\begin{array}{c}\text { Wavelength } \\
{[\AA]}\end{array}$ & $\begin{array}{c}W_{\lambda} \\
{[\mathrm{m} \AA]}\end{array}$ \\
\hline He I & 3888.65 & 148.3 & O II & 4649.14 & 62.4 \\
$\mathrm{He}$ I & 4026.19 & 78.4 & Mg II & 4481.13 & 53.2 \\
$\mathrm{He}$ I & 4471.47 & 125.6 & Si III & 4567.84 & 54.2 \\
$\mathrm{He}$ I & 4471.49 & 136.1 & Si III & 4567.84 & 54.2 \\
$\mathrm{He}$ I & 4471.68 & 51.4 & Fe III & 4137.76 & 50.6 \\
$\mathrm{He}$ I & 4921.93 & 138.1 & Fe III & 4164.73 & 57.2 \\
$\mathrm{He}$ I & 5015.68 & 65.4 & Fe III & 4310.36 & 51.2 \\
$\mathrm{~N}$ II & 3994.99 & 83.5 & Fe III & 4372.82 & 50.0 \\
$\mathrm{~N}$ II & 4241.79 & 57.4 & Fe III & 4419.60 & 55.0 \\
$\mathrm{~N}$ II & 4630.54 & 78.8 & Fe III & 5127.37 & 56.8 \\
$\mathrm{~N}$ II & 5001.13 & 63.0 & & & \\
$\mathrm{~N}$ II & 5001.47 & 60.7 & & & \\
$\mathrm{~N}$ II & 5005.15 & 78.5 & & & \\
\hline
\end{tabular}

just selected lines, so as to account for line blanketing. However the fit is still primarily driven by the Balmer lines with contributions from the strongest metal lines (listed in Table 4). The best fit was found with $T_{\text {eff }}=29370 \mathrm{~K}$ and $\log g=5.48 \mathrm{dex}$, and errors and abundances for the elements that were found to be significant as listed in Table 5. Parameter errors were determined by changing the model in one dimension until the critical $\chi^{2}$-value associated with the probability level at the given number of free parameters was reached. The resulting fit is shown together with the mean spectrum in Fig. 4. While the fit converged with a high rotational velocity for the $\mathrm{sdB}, v \sin i=76 \pm 10 \mathrm{~km} \mathrm{~s}^{-1}$, this value is most likely severely exaggerated due to smearing introduced by the merging of many spectra with poor wavelength calibration, so we do not consider it a real measurement. Note that the higher temperature of the NLTE model is consistent with the typical shift of about $+1000 \mathrm{~K}$ when going from LTE to NLTE (see Heber 2009, and references therein). The abundances show that iron is solar whereas the other elements are subsolar, which is a typical pattern in sdB stars (see e.g. Geier 2013).

\section{High-speed photometric observations}

The pulsations in FBS $0117+396$ were revealed during a short exploratory run with BUSCA. The amplitude spectra corresponding to the light curve in Fig. 1, is plotted in Fig. 2. The most significant peak is found at $2970 \mu \mathrm{Hz}$, with a second lower peak detected at $2610 \mu \mathrm{Hz}$. A possible third peak may be present at $3120 \mu \mathrm{Hz}$.

During the photometric campaign of 2011 we obtained about four hours of data on FBS $0117+396$ on each of six consecutive nights with Ultracam (Dhillon et al. 2007). Additionally, we obtained supporting data from two other observatories on five adjacent nights, four runs with the Merope II imager on
Table 5. Parameters for the fit shown in Fig. 4, with the solar abundances from Asplund et al. (2009) provided for comparison.

\begin{tabular}{llrrrr}
\hline \hline Parameter & Value & $+1 \sigma$ & $-1 \sigma$ & Solar & Unit \\
\hline$T_{\text {eff }}$ & 29370 & 60 & 250 & & $\mathrm{~K}$ \\
$\log g$ & 5.484 & 0.020 & 0.013 & & dex \\
$\log n(\mathrm{He}) / n(\mathrm{H})$ & -2.99 & 0.11 & 0.13 & -1.07 & $\mathrm{dex}$ \\
$\log n(\mathrm{C}) / n(\mathrm{H})$ & -4.92 & 0.75 & 0.57 & -3.57 & dex \\
$\log n(\mathrm{~N}) / n(\mathrm{H})$ & -4.78 & 0.30 & 0.20 & -4.17 & $\mathrm{dex}$ \\
$\log n(\mathrm{O}) / n(\mathrm{H})$ & -4.61 & 0.26 & 0.30 & -3.31 & $\mathrm{dex}$ \\
$\log n(\mathrm{Mg}) / n(\mathrm{H})$ & -5.32 & 0.48 & 0.65 & -4.40 & $\operatorname{dex}$ \\
$\log n(\mathrm{Si}) / n(\mathrm{H})$ & -5.84 & 0.51 & 0.69 & -4.49 & $\operatorname{dex}$ \\
$\log n(\mathrm{Fe}) / n(\mathrm{H})$ & -4.40 & 0.16 & 0.20 & -4.50 & dex \\
\hline
\end{tabular}

the 1.2-m Mercator telescope (Østensen 2010), and three runs with Mexman on the 0.84-m telescope at San Pedro Mártir (SPM) observatory in Mexico, of which one night was too cloudy to be useful (see log of observations in Table 1). The reduced photometric data is shown in Fig. 6. Observing conditions were good on these nights, with clear sky but variable seeing on some nights. While Ultracam was observing in three bands simultaneously, the smaller supporting telescopes used whitelight (unfiltered) observations. White-light data from Ultracam was generated by summing the $r+g+u$ photometry. The shortperiod pulsations are obvious, and the long-period pulsations can also be discerned as excursions with a timescale of about $0.05 \mathrm{~d}$. The orbital frequency is also quite clear, at least when the model plotted with a continuous curve is used as a guide. Since the orbital frequency is close to four cycles per day, it only shifts by 11.6 min every night. Unfortunately, this means we were unable to completely cover the full orbit during the six nights of Ultracam observations.

The photometric data were processed by standard overscan bias-level subtraction and flat-fielding, and the light curves extracted by aperture photometry. For the Ultracam data, we used the provided pipeline (Dhillon et al. 2007), and for the rest of the data we used our own RTP program (Østensen et al. 2001). The extracted photometry was corrected for extinction, using suitable extinction coefficients for the different bands. From these time series, differential photometry was computed by dividing the target data series with that of the sum of six reference stars with brightness comparable to the target. The target and the reference stars are identified in Fig. 5. The Ultracam data were observed using exposure times of $\sim 3$ s for the $r$ and $g$ channels, and twice of that for the $u$ band. The shorter exposure time for the visual bands were required to avoid saturating the red reference stars. For the white-light data shown in Fig. 6 the Ultracam frames for all three bands were coadded to the sampling of the $u$-band.

In order to best extract the $g$-mode pulsations we attempted to orbit correct the differential light curves by fitting a function of the form

$f(t)=A \cos \left(2 \pi \cdot \frac{t-T_{0}}{P}\right)+B \cos \left(4 \pi \cdot \frac{t-T_{0}}{P}\right)+C$

where $P$ is the period, $t$ is the timestamp of each observation, $T_{0}$ is first time of maximum light (zero phase) in the dataset, and $A, B$ and $C$ are the fitting parameters. The form of this function approximates well the irradiation effect in the similar Kepler binaries (Kawaler et al. 2010a), where one only sees the orbital frequency and its first harmonic even in near-continuous datasets spanning a year or more. Using the cosine form with no phase offset ensures that the maximum of the harmonic coincides 


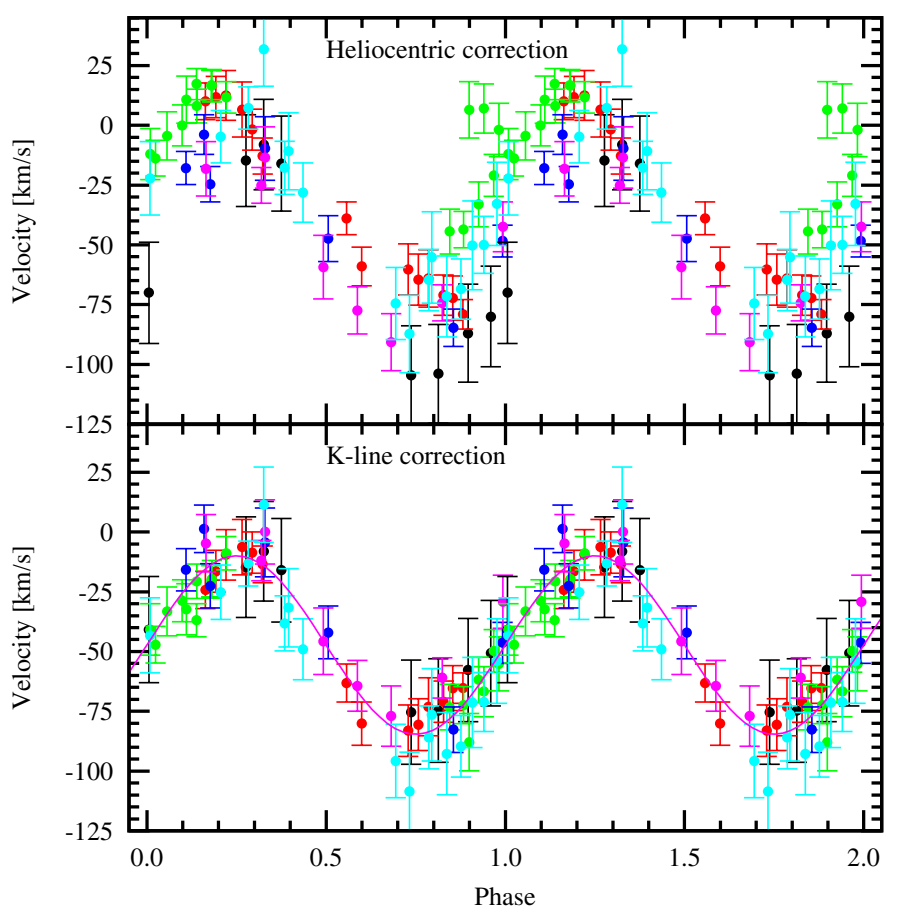

Fig. 3. Radial velocities folded on the best fit orbital period. The upper panel shows the results when using the standard Heliocentric correction, and the lower panel when using the Ca II K-line to correct the zero points. The symbols indicate the origin of the data points, i.e. SDSS (black), WHT 2009 (red) and 2010 (green), NOT 2011/09 (blue) and 2011/11 (magenta), and CAHA (cyan).

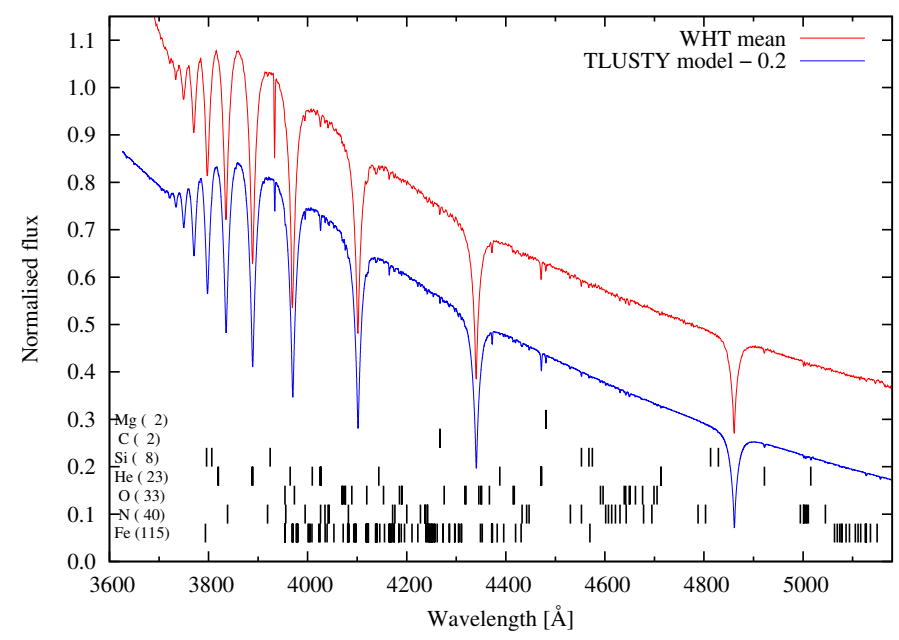

Fig. 4. Sum of the 17 WHT spectra from 2010 after correcting for the orbital velocity. The $\mathrm{S} / \mathrm{N}$ in this mean spectrum peaks at $\sim 350$, and many weak metal lines can be distinguished in addition to the Balmer lines and He I lines at 4472 and $4026 \AA$. The slope of the observed spectrum was corrected by dividing with an instrument response function derived from a flux standard to put it on a $F_{\lambda}$ grid. The absolute units are arbitrary. Shifted down by 0.2 is the model fit computed with TLUSTY/XTGRID. The final parameters for this model fit are given in Table 5.

exactly with the maximum of the orbital period, giving sharp maxima and broad minima, as observed. Thus, when using the period and phase from the radial-velocity solution, with phase corresponding to that of a sine fit (as in Fig. 3), zero phase corresponds to the point in the orbit where the primary is at its closest
Table 6. Detected frequencies, amplitudes and phases.

\begin{tabular}{|c|c|c|c|c|c|}
\hline \multirow[t]{2}{*}{ ID } & \multirow[t]{2}{*}{$\begin{array}{l}\text { Freq. } \\
{[\mu \mathrm{Hz}]}\end{array}$} & \multicolumn{3}{|c|}{$\begin{array}{l}\text { Amplitude } \\
\text { [mma] }\end{array}$} & \multirow[t]{2}{*}{$\begin{array}{l}\text { Phase } \\
\text { [cyc] }\end{array}$} \\
\hline & & $r$ & $g$ & $u$ & \\
\hline$f_{1}$ & $2965.5(2)$ & 7.91(10) & $8.70(10)$ & $12.45(15)$ & $0.045(2)$ \\
\hline$f_{2}$ & $2636.4(2)$ & $2.85(10)$ & $3.05(10)$ & $4.32(15)$ & $0.324(5)$ \\
\hline$f_{A}$ & $115.5(2)$ & $1.67(10)$ & $1.35(10)$ & $2.07(15)$ & $-^{a}$ \\
\hline$f_{B}$ & $171.2(2)$ & $0.80(10)$ & $0.75(10)$ & $1.46(15)$ & $0.79(3)$ \\
\hline$f_{C}$ & $208.5(2)$ & $1.48(10)$ & $1.43(10)$ & $1.91(15)$ & $0.67(1)$ \\
\hline$f_{D}$ & $260.1(2)$ & $0.55(10)$ & $0.42(10)$ & $0.91(15)$ & $0.80(5)$ \\
\hline$f_{E}$ & $264.1(2)$ & $0.82(10)$ & $1.36(10)$ & $0.99(15)$ & $0.50(3)$ \\
\hline$f_{F}$ & $339.6(2)$ & $1.16(10)$ & $1.00(10)$ & $1.37(16)$ & $0.63(2)$ \\
\hline$f_{G}$ & $352.9(2)$ & $0.83(10)$ & $0.94(10)$ & $1.02(16)$ & $0.62(3)$ \\
\hline$f_{H}$ & $359.8(2)$ & $0.57(10)$ & $0.83(10)$ & $0.76(15)$ & $0.08(3)$ \\
\hline
\end{tabular}

Notes. ${ }^{(a)}$ Inconsistent phase in the three bands.

point relative to the observer, which is where we see maximum light for the reflection effect.

Analysis of the white-light data produced consistent frequencies for the two short-period frequencies, but the extended dataset proved to be too noisy at low frequencies to examine the $g$-mode pulsation spectrum. We proceeded to analyse the better quality Ultracam data independently for each photometric band.

The detected frequencies, phases and the amplitudes for the three different bands are listed in Table 6. To derive these we used the classic non-linear-least-squares fitting method described in Vučković et al. (2006). The two short-period modes are trivial to fit, but the messy region below $500 \mu \mathrm{Hz}$ requires some iterations to produce consistent fits due to the forest of one-day alias peaks. We first fitted each band individually, and then proceeded to fix frequencies that showed up consistently in all three bands to the average of the fitting results for the individual bands, in order to produce a ten-frequency fit where the frequencies where fixed and the amplitudes and phases were adjusted. The resulting parameters are listed in Table 6 . There is significant residual power at low frequencies, particularly for the $g$-band that is most likely due to variable sky transparency or other effects not intrinsic to the target, so we did not attempt to fit any signal below $100 \mu \mathrm{Hz}$. The shortest frequency that was consistently found in all bands, $f_{A}=115.5 \mu \mathrm{Hz}$, has a different phase in the $g$-band relative to the two other bands. We cannot say if this is due to an intrinsic frequency being affected by the higher noise at low frequencies in that band, or if that period is a spurious signal due to changing observing conditions. That it has consistent phase in $u$ and $r$ indicates that it is most likely not just noise, but atmospheric effects cannot be ruled out. For pulsators at the hot end of the V1093-Her instability strip, Kepler observations show typical cutoffs at $\sim 100 \mu \mathrm{Hz}$ (Reed et al. 2010a), so we do not expect any of the residuals below that limit to be intrinsic to the star. For the other seven long-period modes the fit converged with consistent phases in all three bands. However, the amplitudes do not always follow the trend one would normally expect for pulsations, where the amplitudes should decrease with wavelength. Instead we see that the $g$-band amplitudes are lower than the $r$-band ones for five of eight of the modes, and the $u$-band amplitude is not the highest for two of the eight, but in all cases these trends are on the $1 \sigma$ level. This may be just a noise artifact, possibly combined with atmospheric effects and dilution from the irradiated secondary, which contaminates the signal more at longer wavelengths. 


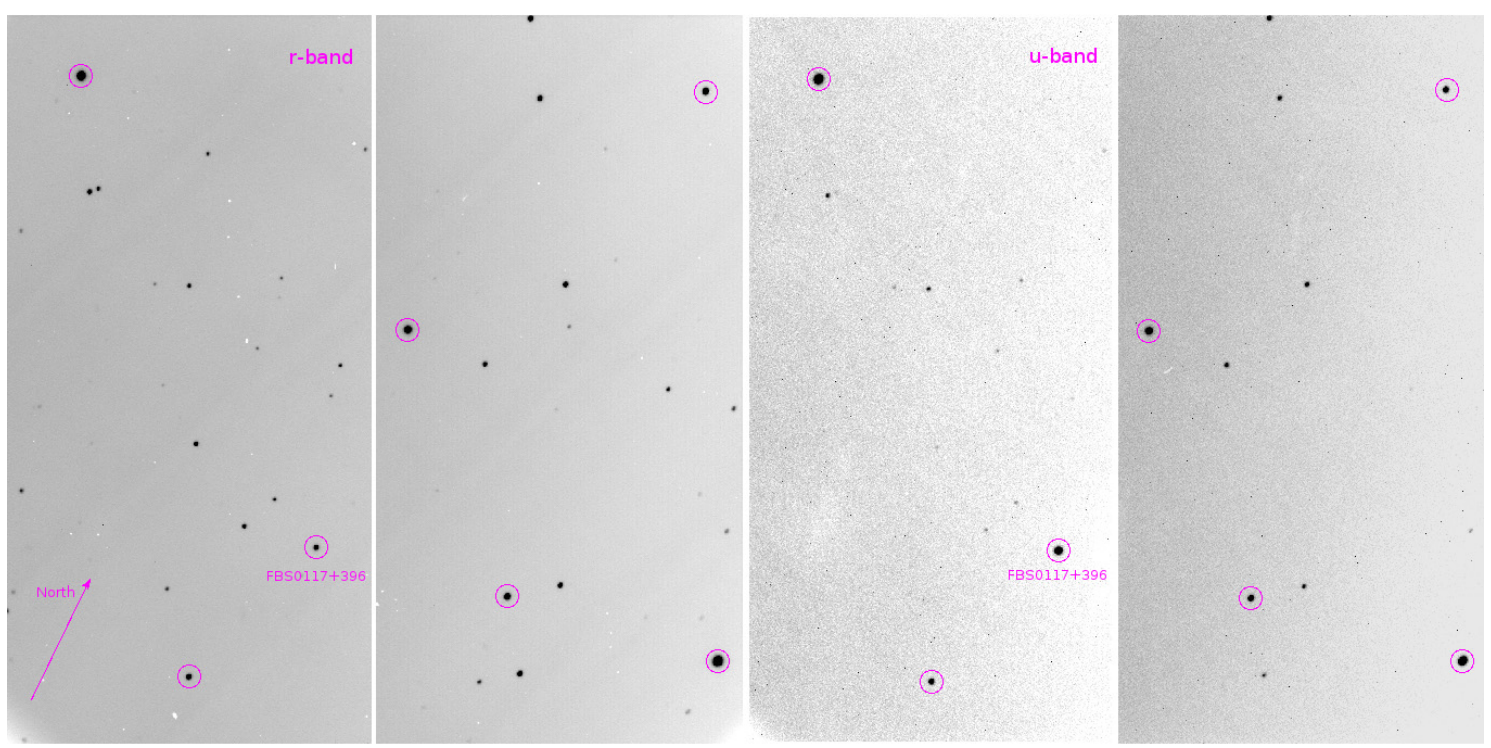

Fig. 5. Ultracam setup frame for the field of FBS $0117+396$. The two images on the left shows average frames from the left and right CCD outputs on the red camera, and the right pair of frames from the $u$-band camera. The target is labeled, and the six reference stars used for the differential photometry are encircled.

For the short-period modes the expected pattern comes out clearly above the noise. The amplitude ratios of the $f_{1}$ and $f_{2}$ are $(r / u, g / u)=(0.64,0.70)$ and $(0.66,0.71)$, which both have slightly higher contrast than ratios computed for a set of evolutionary models with comparable atmospheric parameters from Bloemen et al. (in prep.), but well within the errors on the amplitude measurements. Since $f_{1}$ and $f_{2}$ are too close together to have the same harmonic degree, $\ell$, the simplest assumption is that they are $\ell=0$ and $\ell=1$. If they had the same intrinsic power, visibility would always make the $\ell=0$ the highest amplitude mode, but the slightly higher amplitude ratio for $f_{2}$ than $f_{1}$ is not consistent with this picture. Rather, the amplitude ratio is roughly of the order one would expect if $f_{1}$ is the $\ell=1$ mode.

A Fourier transform of the multi-colour data, before and after prewhitening, is shown in Fig. 7.

\section{Modelling}

In the absence of eclipses it is not possible to determine the orbital inclination from the light curves of $\mathrm{sdB}+\mathrm{dM}$ binaries. In order to investigate the possible ranges that the inclination angle could realistically take, we performed some simple light-curve modelling. As starting assumptions we take the orbital period and the mass function determined in Sect. 3. We assume the mass of the sdB to be that of a canonical EHB $\operatorname{star}\left(M_{1}=0.48 M_{\odot}\right)$ and use the mass-radius (M-R) relationship for M-dwarfs from Baraffe et al. (1998). For each inclination angle, we get from the mass function a value for $M_{2}$, and from the M-R relationship, we get the radius of the secondary. When we plot $M_{2}$ and the corresponding radius, $R_{2}$ as a function of inclination angle, we get the curves in the upper panel of Fig. 8. Inclinations lower than $16^{\circ}$ are excluded as the secondary would fill its Roche lobe.

We can make some simple light curve models just by assuming that each point on the secondary reflects light proportionally to the geometrical fraction of the sky at that point that is covered by the primary (including distance and horizon effects). We only use spherical geometry, taking into account that the primary gets smaller when seen from points that are further away from the substellar point, and bisected by the horizon as one approaches the edge of the irradiated hemisphere. We also take into account limb-darkening according to the square-root law with coefficients from Claret \& Bloemen (2011), as well as eclipses, to produce light curves as shown in the middle panel of Fig. 8. In reality the atmosphere is heated by the UV irradiation from the hot secondary, and in order to estimate colour effects we would have to make assumptions regarding the temperature distribution of the irradiated atmosphere. The problem of degeneracies in such solutions still hamper adequate modelling even in the presence of eclipses, so we will not attempt to produce a complete solution here. Thus, the model light-curves shown here include geometric effects only, and are therefore monochromatic. This is good enough for the eclipse effects, but note that the peak amplitude of the irradiation effect is arbitrary, although we can still make conclusions about how the irradiation effect varies with inclination angle. But it is interesting to note that the amplitude of the irradiation effect is predicted to rise rapidly with decreasing inclination angle, as can be seen in the middle and lower panels of Fig. 8. The amplitude peaks around $22^{\circ}$, due to limb-darkening effects. In models without limb darkening for the secondary, the amplitude does not peak before the Roche limit is reached. Note also that we chose limb-darkening coefficients for the hot side of the secondary to be the same as for the sdB, which is a completely arbitrary choice. In fact, since the atmosphere is inverted (its temperature is highest in the outermost layers), its spectral lines may be in emission as demonstrated for the companion of AA Dor by Vučković et al. (2008), one might expect limb brightening rather than limb darkening, which would give a more extreme inclination/amplitude relationship. To our knowledge, detailed limb-darkening (-brightening) parameters for irradiated M-dwarf atmospheres have not been computed.

The main result here is that the amplitude of the irradiation effect increases with decreasing inclination angle for most of the possible parameter space. This is rather counter-intuitive, but is caused by the constraint on $K_{1}$ from spectroscopy and the relationship between $i$ and and $M_{2}$ and $R_{2}$ that follows. Unfortunately, the absolute value of the amplitude of the reflection effect has too many associated uncertainties to be used to constrain the inclination angle from this effect. We hope that with some modelling of the limb-darkening effects associated with irradiated atmospheres, this situation can be remedied. 
Time [UT]

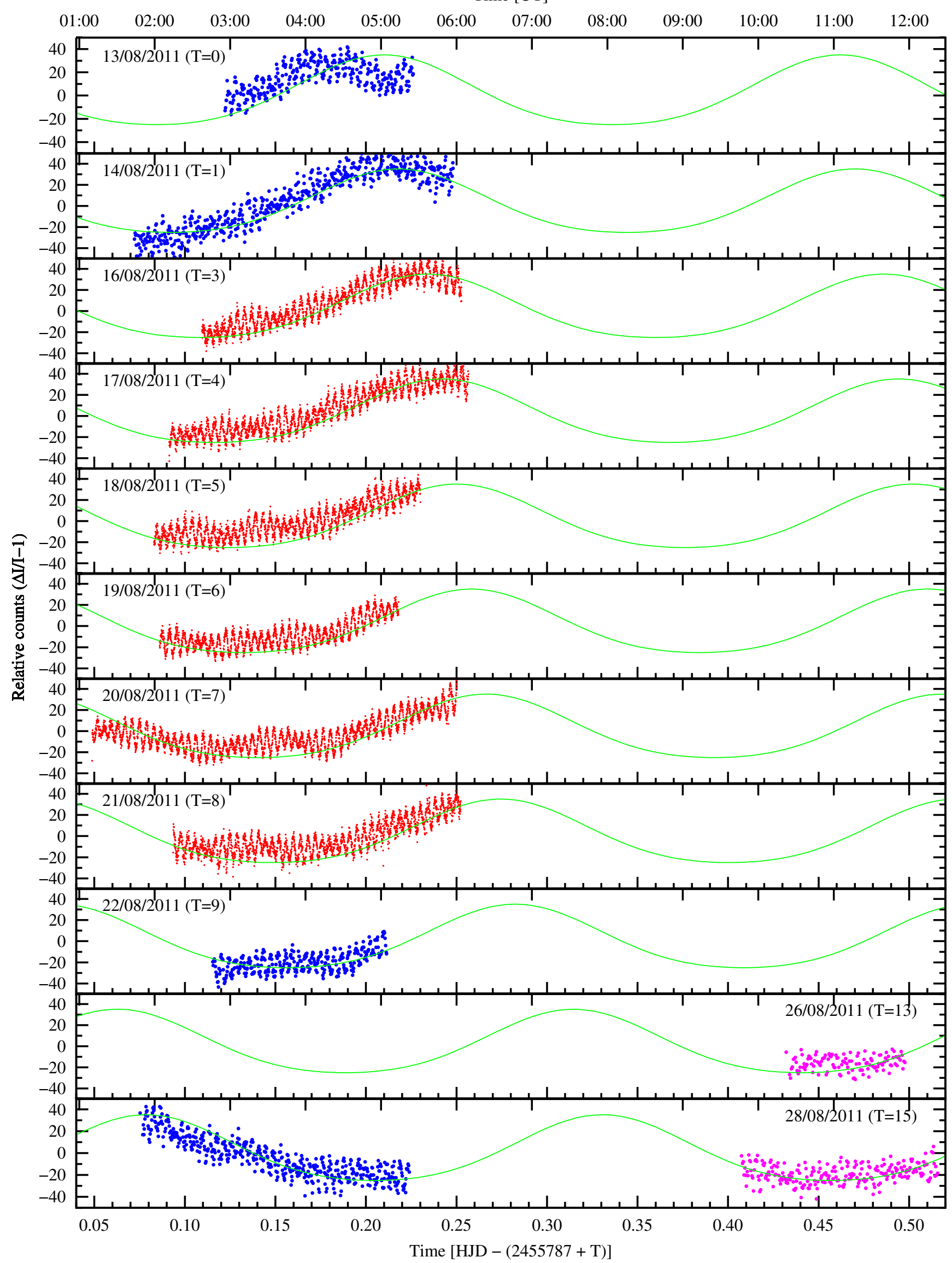

Fig. 6. White-light data for the whole campaign. Red points mark Ultracam, blue points Merope II, and magenta points Mexman data. The solid curve corresponds to the simple model given by Eq. (1), with the period and phase of the radial-velocity solution. 


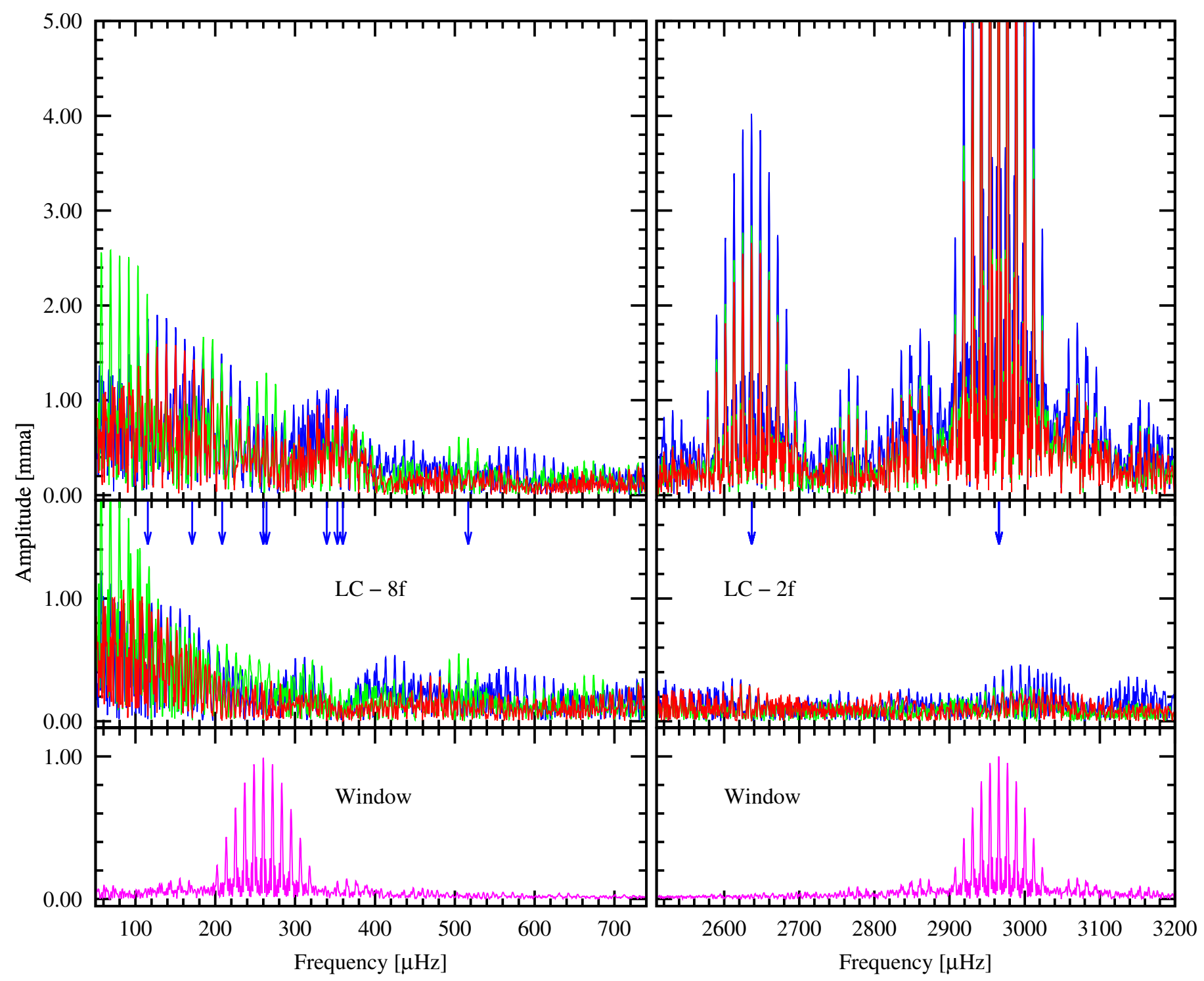

Fig. 7. Amplitude spectrum of the orbit-subtracted Ultracam light curve (LC), for the two frequency regions where pulsations are observed. Upper panel: the amplitude spectra of the three photometric channels (red: $r$-band, green: $g$-band, blue: $u$-band). Middle panel: the same spectra after prewhitening the 10 frequencies listed in Table 6 from the LC. Lower panel: window function for the two frequency regions.

\section{Discussion and conclusions}

Subdwarf-B stars must experience extreme mass loss in order to expel almost their entire hydrogen envelopes on the red-giant branch. Surveys have revealed fractions of short-period binaries that range from 69\% (Maxted et al. 2001) to 39\% (Napiwotzki et al. 2004), depending on how the sample was selected. A large fraction of the remaining stars are binaries in long-period systems, where the mass ratio was inverted during mass transfer, allowing the orbit to expand to exceedingly long periods, but some definitely single stars are known to exist providing an enduring mystery as to their formation (Østensen 2009). Among the short-period binaries, sdB stars with M-dwarf companions are rare compared to systems with unseen companions (presumably WDs); about $1 / 5$ in the compilation of Geier et al. (2011a). Short-period pulsations are only found in $\sim 10 \%$ of $\mathrm{sdB}$ stars that are placed at the hot tip of the EHB (Østensen et al. $2010 \mathrm{~b}$ ), and from that sample of 49 stars plus the recent discoveries, KIC 10139564 (Kawaler et al. 2010b), J08069+1527 (Baran et al. 2011), J20136+0928 (Østensen et al. 2011a), J06398+5156
(Vučković et al. 2012), and the current FBS 0117+396 bringing the total up to 54, we expect about half (27) to be in short-period binaries, and of those $1 / 5$ should be $\mathrm{sdBV}+\mathrm{dM}$ systems. Thus, finding a fourth system still leaves us one short of the predicted five. Although a discrepancy of one is not statistically significant, when considering that many of the 54 short-period sdBVs have still not been carefully checked for radial velocity variations, it is not excluded that more may remain to be discovered.

FBS $0117+396$ falls nicely in the clump of hybrid pulsators in the $T_{\text {eff }} / \log g$ diagram (Fig.9). Up to now it appeared that all the DW-Lyn pulsators that cluster together in the $T_{\text {eff }} / \log g$ diagram were single stars. 2M1938+4603 is an exception, of course, as it is included in the clump of blue points here, but since its pulsation spectrum is quite different from the DW-Lyn stars (it has no high-amplitude $p$-modes) it should perhaps not be termed as such. The V361-Hya star that mingles in on the hot side of the clump is KL UMa (=Feige 48) which is a binary with an unseen companion with a rather long orbital period $(P=9 \mathrm{~h})$ (O'Toole et al. 2004). However, hybrid pulsations have not been detected in this pulsator. 

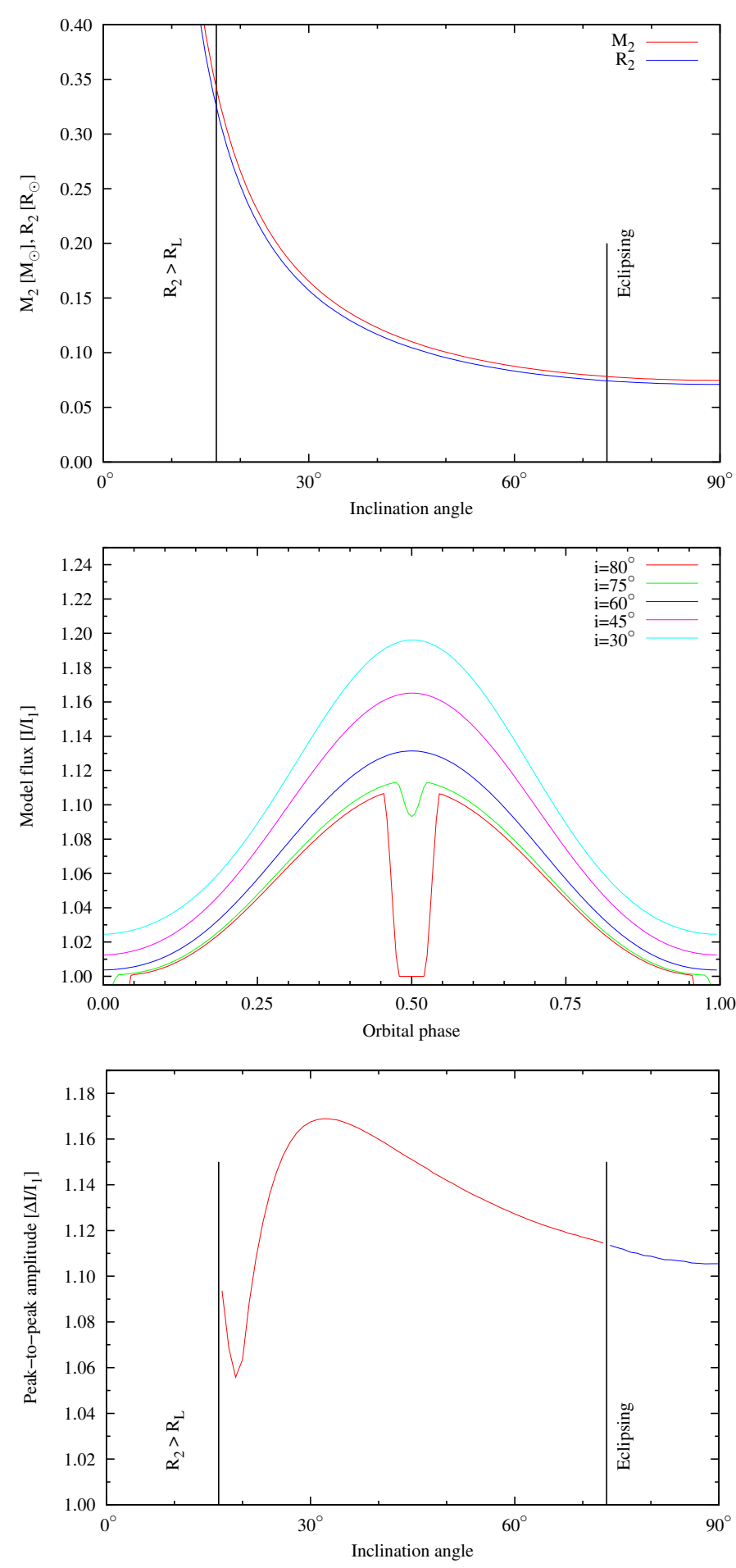

Fig. 8. Upper panel: mass and radius of the secondary component as a function of inclination angle, as inferred by the mass function and the $\mathrm{M} / \mathrm{R}$ relationship for M-dwarfs. Middle panel: model light-curves for a few inclination angles, computed using the radius relationship from the upper panel. $I_{1}$ is the model flux of the primary alone, and $I$ is the total model flux from the system. Lower panel: peak-to-peak amplitude of the reflection effect for all inclination angles. $\Delta I$ is the difference in model flux at phase 0 and phase 0.5 , when ignoring eclipses.

FBS $0117+396$ is particularly interesting as its orbital period at $\sim 6 \mathrm{~h}$ is longer than that of the stars that are known to be tidally locked (NY Vir; $P=2.4 \mathrm{~h}, 2 \mathrm{M} 1938+4603 ; P=3 \mathrm{~h}$ ) and the Kepler binaries of Pablo et al. (2011) which at $P \approx 10 \mathrm{~h}$ are

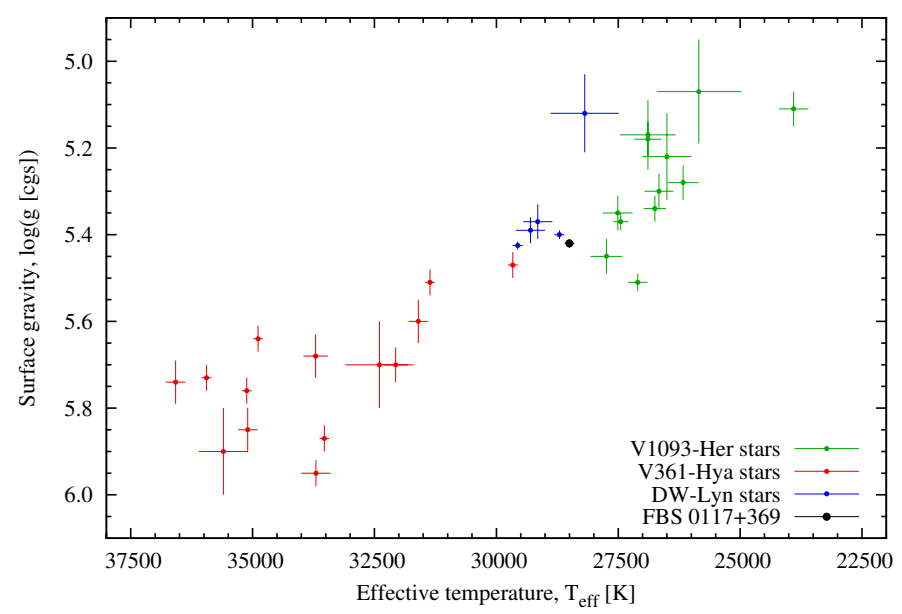

Fig. 9. $T_{\text {eff }} / \log g$ diagram. FBS $0117+396$ falls neatly with the close group of hybrid (DW-Lyn type) sdBV stars. The V1093-Her stars are the pulsators in the Kepler sample with parameters from Østensen et al. (2010c, 2011b), and the other stars are summarised in Østensen et al. (2010b), but we have only included those that have been fitted on the same LTE grid as described in Sect. 3.1.

not. But whether or not FBS $0117+396$ is in a tidally locked orbit cannot be determined from the current data. The observed velocity required for a locked state depends on the inclination angle, and for $i=45^{\circ}$ and $R_{1}=0.22 R_{\odot}$, we would expect $v \sin i=44 \mathrm{~km} \mathrm{~s}^{-1}$, which is not detectable in our low $\mathrm{S} / \mathrm{N}$ individual spectra that has a resolution of $\sim 75 \mathrm{~km} \mathrm{~s}^{-1}$ at best. It might have been detectable in some of our mean spectra if we could have added up the individual observations while correcting for the orbital Doppler shifts, thereby beating down the noise. However, the problems with the wavelength calibration discussed in Sect. 3 hobbles this approach. Furthermore, the pulsation spectrum is too simple to give any constraints on the rotation from rotationally split multiplets. Thus, we are not able to constrain the value of the rotation from the available data. A high-resolution spectrum of FBS 0117+396 would help with resolving this issue.

Acknowledgements. Based on observations collected at the Nordic Optical Telescope (NOT), the William Herschel Telescope (WHT) and the Mercator Telescope, all on the island of La Palma, Spain, and at the Centro Astrónomico Hispano Alemán (CAHA) at Calar Alto, Spain, and at the Observatorio Astronómico Nacional (OAN) at San Pedro Mártir, Mexico. We gratefully acknowledge using spectroscopic data from the database of the Sloan Digital Sky Survey (SDSS). The research leading to these results has received funding from the European Research Council under the European Community's Seventh Framework Programme (FP7/2007-2013)/ERC grant agreement №227224 (PROSPERITY), from the Research Council of KU Leuven grant agreement GOA/2008/04, and from the Fund for Scientific Research of Flanders (FWO), grant agreements G.0332.06 and G.0470.07, and G.0C31.13. The CCD of the Merope II camera is part of a set developed by E2V in the framework of the Eddington space mission project and are owned by the European Space Agency; they were offered on permanent loan to the Institute of Astronomy of KU Leuven, Belgium, with the aim to build and exploit an instrument for asteroseismology research to be installed at the Mercator telescope. Conny Aerts is grateful to Giuseppe Sarri and Fabio Favata for their support and help in the practical implementation of the ESA loan agreement with K. U. Leuven. S.G., A.T., E.Z. and travel to Calar Alto were supported by the Deutsche Forschungsgemeinschaft (DFG) through grants HE1356/49-1, HE1356/45-1 HE1356/45-2, HE1356/53-1, and HE1356/64-1. V.S. acknowledges funding by the Deutsches Zentrum für Luft- und Raumfahrt (grant 50 OR 1110) and by the Erika-Giehrl-Stiftung. L.F.M. acknowledges financial support from the UNAM under grant PAPIIT 104612. S.B. is supported by the Foundation for Fundamental Research on Matter (FOM), which is part of the Netherlands Organisation for Scientific Research (NWO). 


\section{References}

Abrahamian, H. V., Lipovetski, V. A., Mickaelian, A. M., \& Stepanian, J. A. 1990, Astrofizika, 33, 213

Aerts, C., Christensen-Dalsgaard, J., \& Kurtz, D. W. 2010, Asteroseismology (Springer)

Asplund, M., Grevesse, N., Sauval, A. J., \& Scott, P. 2009, ARA\&A, 47, 481

Baraffe, I., Chabrier, G., Allard, F., \& Hauschildt, P. H. 1998, A\&A, 337, 403

Baran, A. S., Gilker, J. T., Reed, M. D., et al. 2011, MNRAS, 413, 2838

Barlow, B. N., Wade, R. A., Liss, S. E., Østensen, R. H., \& Van Winckel, H. 2012, ApJ, 758, 58

Charpinet, S., van Grootel, V., Reese, D., et al. 2008, A\&A, 489, 377

Claret, A., \& Bloemen, S. 2011, A\&A, 529, A75

Copperwheat, C. M., Morales-Rueda, L., Marsh, T. R., Maxted, P. F. L., \& Heber, U. 2011, MNRAS, 415, 1381

Deca, J., Marsh, T. R., Østensen, R. H., et al. 2012, MNRAS, 421, 2798

Dhillon, V. S., Marsh, T. R., Stevenson, M. J., et al. 2007, MNRAS, 378, 825

Geier, S. 2013, A\&A, 549, A110

Geier, S., Hirsch, H., Tillich, A., et al. 2011a, A\&A, 530, A28

Geier, S., Maxted, P. F. L., Napiwotzki, R., et al. 2011b, A\&A, 526, A39

Geier, S., Schaffenroth, V., Drechsel, H., et al. 2011c, ApJ, 731, L22

Geier, S., Schaffenroth, V., Hirsch, H., et al. 2012, in Fifth Meeting on Hot Subdwarf Stars and Related Objects, eds. D. Kilkenny, C. S. Jeffery, \& C. Koen, ASP Conf. Ser., 452, 129

Green, E. M., Fontaine, G., Reed, M. D., et al. 2003, ApJ, 583, L31

Heber, U. 2009, ARA\&A, 47, 211

Heber, U., Reid, I. N., \& Werner, K. 2000, A\&A, 363, 198

Hubeny, I., \& Lanz, T. 1995, ApJ, 439, 875

Kawaler, S. D., Reed, M. D., Østensen, R. H., et al. 2010a, MNRAS, 409, 1509

Kawaler, S. D., Reed, M. D., Quint, A. C., et al. 2010b, MNRAS, 409, 1487

Kawka, A., Pigulski, A., O'Toole, S., et al. 2012, in Fifth Meeting on Hot Subdwarf Stars and Related Objects, eds. D. Kilkenny, C. S. Jeffery, \& C. Koen, ASP Conf. Ser., 452, 121

Kilkenny, D., Koen, C., O’Donoghue, D., \& Stobie, R. S. 1997, MNRAS, 285, 640

Kilkenny, D., O’Donoghue, D., Koen, C., Lynas-Gray, A. E., \& van Wyk, F. 1998, MNRAS, 296, 329

Kilkenny, D., Koen, C., \& Worters, H. 2010, MNRAS, 404, 376

Koen, C. 2009, MNRAS, 395, 979

Koen, C., O’Donoghue, D., Kilkenny, D., Stobie, R. S., \& Saffer, R. A. 1999, MNRAS, 306, 213

Lisker, T., Heber, U., Napiwotzki, R., et al. 2005, A\&A, 430, 223
Maxted, P. F. L., Heber, U., Marsh, T. R., \& North, R. C. 2001, MNRAS, 326, 1391

Napiwotzki, R., Karl, C. A., Lisker, T., et al. 2004, Ap\&SS, 291, 321

Németh, P., Kawka, A., \& Vennes, S. 2012, MNRAS, 427, 2180

Østensen, R. H. 2009, Commun. Asteroseismol., 159, 75

Østensen, R. H. 2010, Astron. Nachr., 331, 1026

Østensen, R. H., \& van Winckel, H. 2011, in Evolution of Compact Binaries, eds. L. Schmidtobreick, M. R. Schreiber, \& C. Tappert, ASP Conf. Ser., 447, 171

Østensen, R. H., \& Van Winckel, H. 2012, in Fifth Meeting on Hot Subdwarf Stars and Related Objects, eds. D. Kilkenny, C. S. Jeffery, \& C. Koen, ASP Conf. Ser., 452, 163

Østensen, R., Solheim, J.-E., Heber, U., et al. 2001, A\&A, 368, 175

Østensen, R. H., Green, E. M., Bloemen, S., et al. 2010a, MNRAS, 408, L51

Østensen, R. H., Oreiro, R., Solheim, J., et al. 2010b, A\&A, 513, A6

Østensen, R. H., Silvotti, R., Charpinet, S., et al. 2010c, MNRAS, 409, 1470

Østensen, R. H., Pápics, P. I., Oreiro, R., et al. 2011a, ApJ, 731, L13

Østensen, R. H., Silvotti, R., Charpinet, S., et al. 2011b, MNRAS, 414, 2860

O’Toole, S. J., Heber, U., \& Benjamin, R. A. 2004, A\&A, 422, 1053

Pablo, H., Kawaler, S. D., \& Green, E. M. 2011, ApJ, 740, L47

Pablo, H., Kawaler, S. D., Reed, M. D., et al. 2012, MNRAS, 422, 1343

Reed, M. D., \& Stiening, R. 2004, PASP, 116, 506

Reed, M., Kawaler, S. D., Østensen, R. H., et al. 2010a, MNRAS, 409, 1496

Reed, M. D., Terndrup, D. M., Østensen, R., et al. 2010b, Ap\&SS, 329, 83

Reif, K., Bagschik, K., de Boer, K. S., et al. 1999, in Proc. SPIE 3649, eds. M. M. Blouke, \& G. M. Williams, 109

Schlegel, D. J., Finkbeiner, D. P., \& Davis, M. 1998, ApJ, 500, 525

Skrutskie, M. F., Cutri, R. M., Stiening, R., et al. 2006, AJ, 131, 1163

Stoughton, C., Lupton, R. H., Bernardi, M., et al. 2002, AJ, 123, 485

Van Grootel, V., Charpinet, S., Brassard, P., Fontaine, G., \& Green, E. M. 2013, A\&A, 553, A97

Vos, J., Østensen, R. H., Degroote, P., et al. 2012, A\&A, 548, A6

Vučković, M., Kawaler, S. D., O’Toole, S., et al. 2006, ApJ, 646, 1230

Vučković, M., Aerts, C., Østensen, R., et al. 2007, A\&A, 471, 605

Vučković, M., Østensen, R., Bloemen, S., Decoster, I., \& Aerts, C. 2008, in Hot Subdwarf Stars and Related Objects, eds. U. Heber, C. S. Jeffery, \& R. Napiwotzki, ASP Conf. Ser., 392, 199

Vučković, M., Østensen, R. H., Acke, B., et al. 2012, in Fifth Meeting on Hot Subdwarf Stars and Related Objects, eds. D. Kilkenny, C. S. Jeffery, \& C. Koen, ASP Conf. Ser., 452, 257 